\title{
A Fiberized Metamaterial Device for Ultrafast Control of Coherent Optical Signals
}

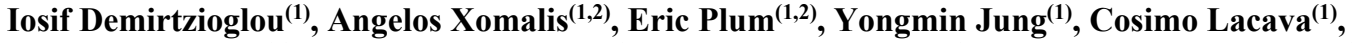 \\ Kevin F. MacDonald ${ }^{(1,2)}$, Periklis Petropoulos ${ }^{(1)}$, David J. Richardson ${ }^{(1)}$, and Nikolay I. Zheludev ${ }^{(1,2,3)}$ \\ (1) Optoelectronics Research Centre, University of Southampton, Southampton, SO17 1BJ, UK \\ (2) Centre for Photonic Metamaterials, University of Southampton, Southampton, SO17 1BJ, UK \\ (3) Centre for Disruptive Photonic Technologies, School of Physical and Mathematical Sciences and the Photonics Institute, Nanyang \\ Technological University, Singapore, 637371 \\ E-mail address: id3n14@soton.ac.uk
}

\begin{abstract}
We demonstrate selective transmission and absorption of 1-ps pulses, pulse shaping and 1 -ps dark pulse generation in a fiber-optic device based on a plasmonic metamaterial, providing an example of all-optical signal processing with $\mathrm{THz}$ bandwidth.

OCIS codes: (060.2310) Fiber optics; (160.3918) Metamaterials; (230.1150) All-optical devices
\end{abstract}

\section{Introduction}

All-optical processes that rely on manipulation of electrons offer operation speeds that are much faster than the bandwidth of common optical communication signals, and as such they are very attractive for the implementation of switching devices. Typical ultrafast all-optical switches make use of either nonlinear processes, which require relatively high power (e.g. [1-4]), or rely on interference. An interference mechanism that has been proposed recently for nm-scale all-optical data processors, is based on coherent absorption and coherent transmission of signals that interfere in an ultrathin film [5]. Demonstrations of such coherent control of light with light have previously been presented on metamaterial structures in free space [6].

In this work, we exploit coherent absorption and coherent transparency to control picosecond-long optical pulses in a fully packaged, fiber-pigtailed metadevice, which consists of a plasmonic nanostructure attached to an optical fiber. We investigate the coherent interaction of pulses of different widths, which allows shaping of the pulse envelope, including the formation of picosecond scale dark pulses via coherent absorption. This represents, to our knowledge, the highest speed of operation demonstrated in a fiberized metadevice throughout the C-band.

\section{Principle and device fabrication}

The operation of the fiberized metadevice is based on the interaction of two coherent counter-propagating optical signals on a nanostructured film of substantially subwavelength thickness. Figure 1a shows a schematic representation of the electric field of two optical signals propagating in opposite directions. The response of an absorptive thin film placed normal to the propagation axis depends on its position relative to the standing wave formed by the two waves. When it is placed at a node of the standing wave, where the two beams add destructively resulting in zero electric field, the thin film becomes effectively transparent as it cannot interact with the wave. In contrast, when it is placed at an anti-node, where constructive interference of the electric field occurs, it interacts with the wave and shows strong absorption. In contrast to common interference phenomena that redirect light between different outputs (e.g. when the states of constructive and destructive interference of two waves are directed to the two outputs of a fiber coupler), here, interference controls absorption of light and results in two counterpropagating output signals whose respective states are identical.

Coherent absorption was realized on a 70-nm-thick gold film, perforated with an array of asymmetrically split ring apertures. The design of the plasmonic nanostructure aimed for equal levels of transmission and reflection to allow ideally for coherent control of absorption from $0 \%$ to $100 \%$, along with $50 \%$ single-beam absorption which is the limit for planar materials. The final geometry of the fabricated gold film is illustrated in the scanning electron microscopy (SEM) images of Fig. 1b. Its fabrication and packaging in a fiberized device included thermal evaporation of gold and focused ion beam milling on the cleaved end of a single-mode polarization-maintaining (PM) telecommunications fiber, such that the symmetry axis of the metamaterial was aligned to the slow axis of the fiber. Coupling to a second PM fiber was provided by a set of microcollimator lenses, and the two fibers were packaged to form the pigtailed metadevice shown in Fig. 1b. A measurement of the single-beam transmission and reflection of the device revealed an asymmetric behavior due to the asymmetric structure of the device as well as fabrication and packaging imperfections. For a single input signal, $\mathrm{I}_{\mathrm{a}}\left(\right.$ or $\mathrm{I}_{\mathrm{b}}$ ), the fabricated device transmitted $24 \%$ (24\%), reflected 18\% (8\%) and introduced 58\% (68\%) losses; note these values also include any losses introduced by the packaging. 
(a)

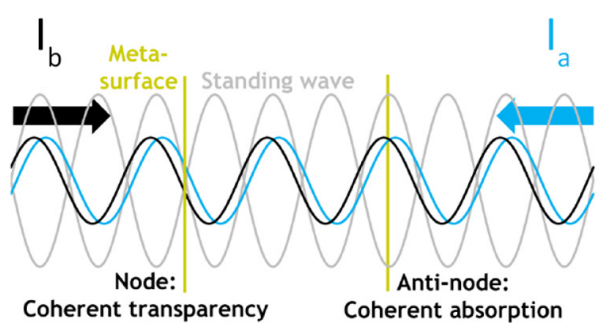

(b)

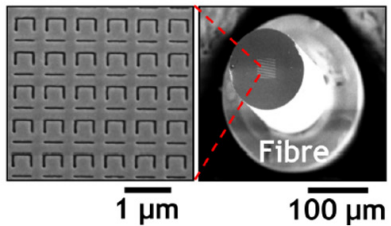

(c)

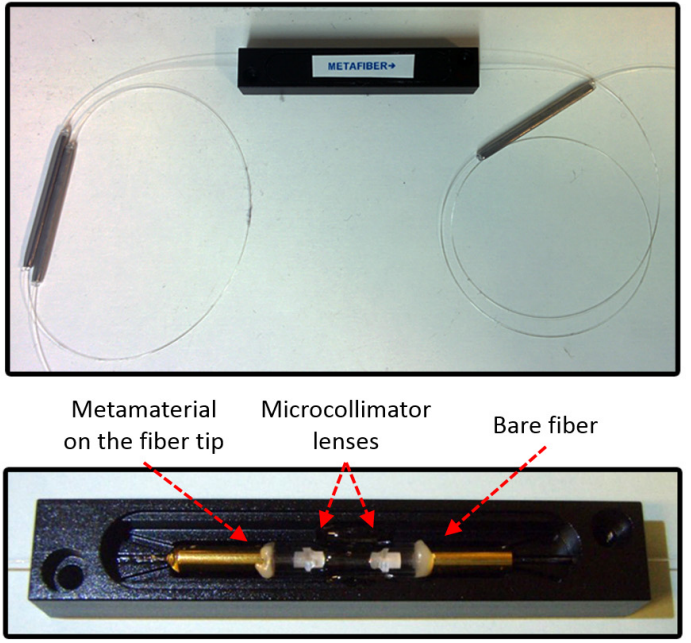

Fig. 1. (a) Standing wave formation and its interaction with a metasurface, (b) SEM images of the 70-nm thick metasurface, (c) Top-view photographs of the fiberized metadevice (bottom: inside view).

\section{Experimental setup and results}

The physical mechanism of absorption in the thin film is based on plasmonic excitation, therefore its switching speed is linked to the plasmon relaxation time in gold. This means that the bandwidth of the coherent absorption mechanism will be in the range of tens of $\mathrm{THz}$ [6]. Our experiments were carried out using 1-ps pulses produced by a $10-\mathrm{GHz}$ broadband frequency comb generator (OptoComb LP-5011F1) modulating a CW laser in the region of $1550 \mathrm{~nm}$ (Fig. 2). The optical pulses were amplified and split along two paths in order for them to be launched into the metadevice from opposite sides. A tunable free-space optical delay line (ODL) was used to control the relative delay of the pulses in the two paths, and to ensure that they overlapped on the surface of the metadevice. Two variable optical attenuators (VOAs) were used to set the pulse peak powers of the two branches of the interferometer to equal levels. The output signal was extracted by means of fiber circulators connected to the pigtails of the metadevice. With the exception of the ODL, all components used in the set-up were PM. Therefore, a polarization controller (PC) along with a polarization beam splitter (PBS) were used to ensure the correct polarization was launched into the device. The maximum average input power launched to the device was $607 \mu \mathrm{W}$, which corresponded to a maximum measured pulse energy of $17.4 \mathrm{fJ}$. The output $\mathrm{I}_{\mathrm{d}}$ of the device was measured on an optical sampling oscilloscope (EXFO PSO-102).

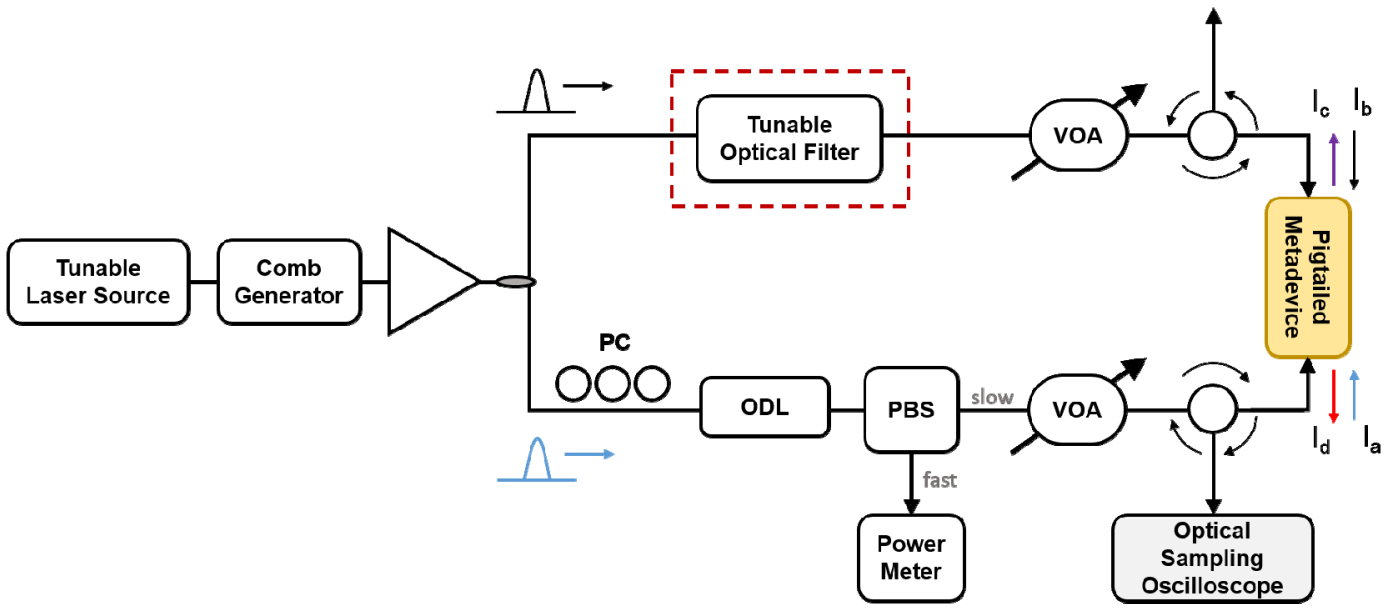

Fig. 2. Experimental setup for measuring all-optical modulation of trains of picosecond pulses within the metadevice.

Without any active stabilization of the two optical path lengths, the phase difference between the beams was free to change over time due to environmental instabilities. By means of the ODL, we overlapped the pulses on the 
metadevice and observed as all possible states were traversed over a timescale in the order of seconds. We recorded this behavior for the states of coherent transparency and coherent absorption and the results are shown in Fig. 3 (top-left). The measured peak power for coherent absorption was $15 \%(-8.2 \mathrm{~dB})$ of the peak power for coherent transparency when the powers of the two incoming pulses was set to be equal to each other. This contrast could be increased further by optimizing the single-beam transmission, reflection and loss characteristics of the device. It is noted that coherent transparency and absorption can be treated as implementations of logical AND and XOR operators, respectively, provided that proper thresholding and phase control are applied.

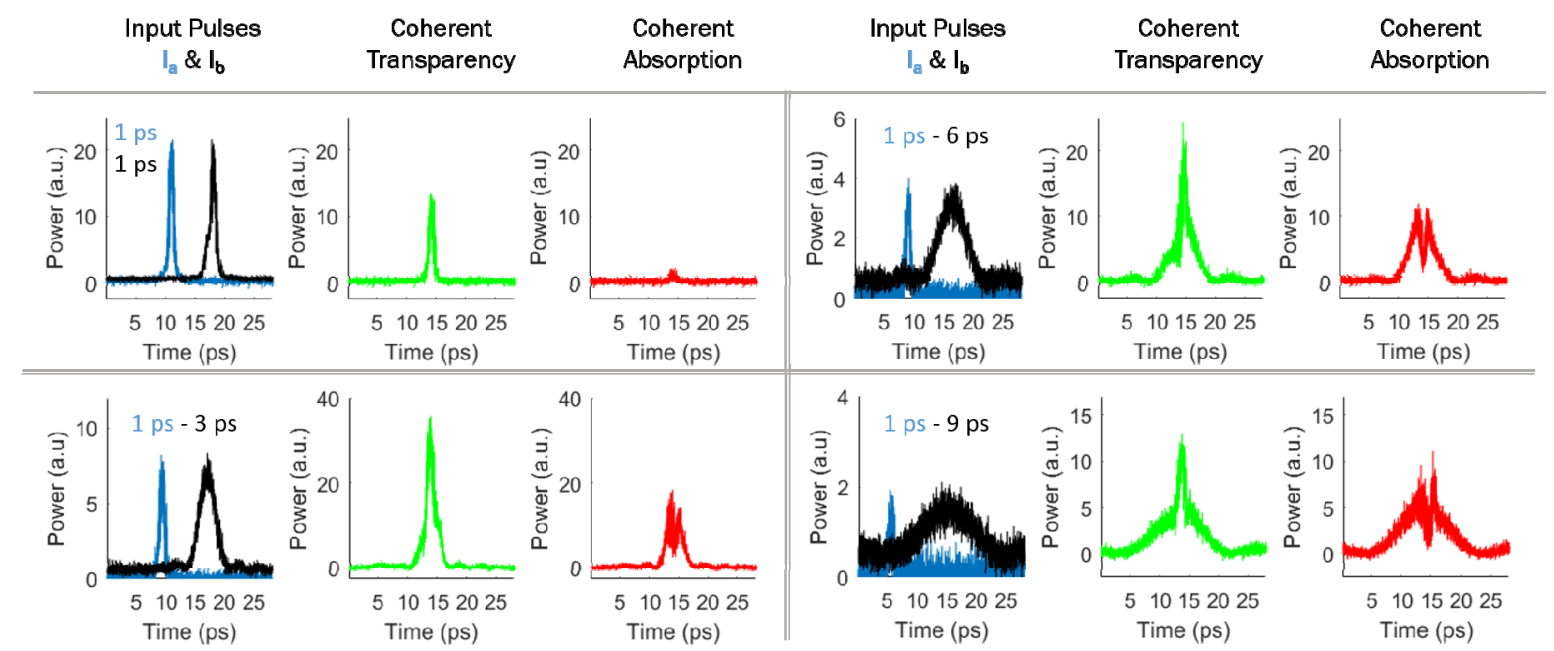

Fig. 3. Measured pulses at the inputs $I_{a}, I_{b}$ and output $I_{d}$ of the metadevice for different pulse widths of input $I_{b}$. Measurements of the output $I_{d}$ for the cases of 3, 6 and 9 ps input pulses are recorded after optical amplification.

To further explore the potential applications of the metadevice in all-optical control of signals, we studied the interaction between pulses of different widths. By spectral filtering of a portion of the comb using a tunable-bandwidth optical filter, we controllably broadened the pulses propagating in one arm of the interferometer. The interaction between the original pulse and a broadened one is depicted in Fig. 3 for three different pulse widths, namely 3 ps, 6 ps and 9 ps. In all cases, by means of the VOA, we adjusted the peak power of the 1-ps pulses in the second arm to match that of the broadened pulses. The coherent transparency state resulted in constructive addition of the two pulse shapes, increasing the peak power by more than a factor of two. In contrast, coherent absorption resulted in the formation of dark pulses, where the power was reduced to $<30 \%$ compared to the part of the broader pulse that did not interact with the shorter one.

\section{Conclusion}

A fiberized metadevice for coherent all-optical signal processing was designed and fabricated. Shaping and modulation of picosecond pulses including the creation of dark pulses was presented in the C-band at sub-milliwatt average powers. The observed coherent transmission and coherent absorption of 1 ps pulses demonstrates the ability to process optical signals with a minimum bandwidth of $1 \mathrm{THz}$, and since the modulation is based on linear absorption in a plasmonic absorber, we anticipate a potential bandwidth of tens of THz.

\section{Acknowledgments}

This work has been supported by EPSRC, UK (grants EP/M009122/1 and EP/L00044X/1) and the MOE Singapore (grant MOE2011-T3-1-005). The data for this work is accessible through the University of Southampton Institutional Research Repository: https://doi.org/10.5258/SOTON/D0275.

\section{References}

[1] K. Nozaki et al., "Sub-femtojoule all-optical switching using a photonic-crystal nanocavity," Nat. Photonics 4, 477-483 (2010).

[2] V. R. Almeida et al., "All-optical control of light on silicon chip," Nature 431, 1081-1084 (2004).

[3] A. E. Nikolaenko et al., "Nonlinear graphene metamaterial," Appl. Phys. Lett. 100, 17-19 (2012).

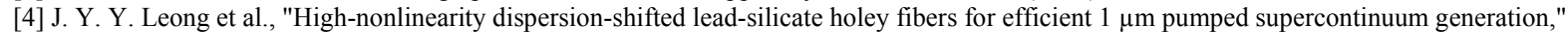

J. Lightwave Technol. 24, 183-190 (2006).

[5] X. Fang et al., "Controlling light with light using coherent metadevices: all-optical transistor, summator and invertor," Light Sci. Appl. 4, e292 (2015).

[6] V. Nalla et al., "11-Fs Dark Pulses Generated Via Coherent Absorption in Plasmonic Metamaterial," Opt. Express 14, 868-870 (2016). 